\title{
BOUNDEDNESS AND VANISHING OF SOLUTIONS FOR A FORCED DELAY DYNAMIC EQUATION
}

\author{
DOUGLAS R. ANDERSON
}

Received 30 March 2006; Revised 10 July 2006; Accepted 14 July 2006

We give conditions under which all solutions of a time-scale first-order nonlinear variable-delay dynamic equation with forcing term are bounded and vanish at infinity, for arbitrary time scales that are unbounded above. A nontrivial example illustrating an application of the results is provided.

Copyright (c) 2006 Douglas R. Anderson. This is an open access article distributed under the Creative Commons Attribution License, which permits unrestricted use, distribution, and reproduction in any medium, provided the original work is properly cited.

\section{Delay dynamic equation with forcing term}

Following Hilger's landmark paper [8], a rapidly expanding body of literature has sought to unify, extend, and generalize ideas from discrete calculus, quantum calculus, and continuous calculus to arbitrary time-scale calculus, where a time scale is simply any nonempty closed set of real numbers. This paper illustrates this new understanding by extending some continuous results from differential equations to dynamic equations on time scales, thus including as corollaries difference equations and $q$-difference equations. Throughout this work, we consider the nonlinear forced delay dynamic equation

$$
x^{\Delta}(t)=-p(t) f(x(\tau(t)))+r(t), \quad t \in\left[t_{0}, \infty\right)_{\mathbb{T}}, t_{0} \geq 0
$$

where $\mathbb{T}$ is a time scale unbounded above, $f: \mathbb{R} \rightarrow \mathbb{R}$ is continuous, and the functions $p$ : $\mathbb{T} \rightarrow(0, \infty)$ and $r: \mathbb{T} \rightarrow \mathbb{R}$ are both right-dense continuous. Moreover, the variable delay $\tau: \mathbb{T} \rightarrow \mathbb{T}$ is increasing with $\tau(t) \leq t$ for all $t \in\left[t_{0}, \infty\right)_{\mathbb{T}}$ such that $\lim _{t \rightarrow \infty} \tau(t)=\infty$. The initial function associated with (1.1) takes the form $x(t)=\psi(t)$ for $t \in\left[\tau\left(t_{0}\right), t_{0}\right]$, where $\psi$ is rd-continuous on $\left[\tau\left(t_{0}\right), t_{0}\right]$. Equation (1.1) is studied extensively by Qian and Sun $[13]$ in the case when $\mathbb{T}=\mathbb{R}$. See also related discussions on unforced delay equations by Matsunaga et al. [12] in the continuous case, and by Erbe et al. [6] or Zhang and Yan [14] 
2 Forced delay dynamic equation

in the discrete case. Other papers on delay dynamic equations include [1-3]. For more on dynamic equations on time scales, skip ahead to the appendix, Section 5, or consult the recent texts by Bohner and Peterson $[4,5]$. To clarify some notation, take $\tau^{-1}(t):=$ $\sup \{s: \tau(s) \leq t\}, \tau^{-(n+1)}(t)=\tau^{-1}\left(\tau^{-n}(t)\right)$ for $t \in\left[\tau\left(t_{0}\right), \infty\right)_{\mathbb{T}}$, and $\tau^{n+1}(t)=\tau\left(\tau^{n}(t)\right)$ for $t \in\left[\tau^{-3}\left(t_{0}\right), \infty\right)_{\mathbb{T}}$. By our choice of the delay $\tau$, there exists large $T \in \mathbb{T}$ such that $\tau(t) \geq t_{0}$ and $\tau^{2}(t) \leq \tau(t) \leq t \leq \tau^{-1}(\sigma(t))$ for all $t \geq T$. In addition, we always suppose that

(H1) the continuous function $f$ satisfies $|f(x)|<|x|$ and $x f(x)>0$ for $x \neq 0$, with

$$
f^{\dagger}(x):=\max \left\{\sup _{0 \leq u \leq|x|} f(u), \sup _{0 \leq u \leq|x|}(-f(-u))\right\} \quad x \in \mathbb{R} ;
$$

(H2) using the delay $\tau$, the forcing function $r$ satisfies

$$
\sum_{n=0}^{\infty} \int_{\tau^{1-n}\left(t_{0}\right)}^{\infty}|r(s)| \Delta s<\infty
$$

(H3) the coefficient function $p$ satisfies

$$
\int_{\tau(t)}^{\sigma(t)} p(s) \Delta s \leq \lambda \quad \forall t \in\left[t_{0}, \infty\right)_{\mathbb{T}}, \quad \int_{t_{0}}^{\infty} p(s) \Delta s=\infty
$$

where

$$
\lambda:=\frac{3}{2}+\frac{1}{2} \frac{\inf \{\mu(t): t \in \mathbb{T}\}}{\sup \left\{\tau^{-1}(\sigma(t))-t: t \in \mathbb{T}\right\}}
$$

it is understood that $\lambda=3 / 2$ if either $\inf \{\mu(t)\}=0$ or $\sup \left\{\tau^{-1}(\sigma(t))-t\right\}=\infty$.

\section{Background lemmas}

We will need Lemma 2.1 in the proof of Lemma 2.2.

Lemma 2.1 [1, Lemma 2.1]. For a right-dense continuous function $p: \mathbb{T} \rightarrow \mathbb{R}$ and points $a, t \in \mathbb{T}$,

$$
\int_{a}^{t}\left(p(s) \int_{a}^{\sigma(s)} p(u) \Delta u\right) \Delta s=\frac{1}{2}\left(\int_{a}^{t} p(s) \Delta s\right)^{2}+\frac{1}{2} \int_{a}^{t} \mu(s) p^{2}(s) \Delta s .
$$

Lemma 2.2. Assume (H1), (H2), (H3) hold. Let $x$ be a solution of (1.1), and assume there exists $t_{1} \in\left(\tau^{-2}(T), \infty\right)_{\mathbb{T}}$ such that $\tau^{2}\left(t_{1}\right) \geq t_{0}$ and $x\left(t_{1}\right) x^{\sigma}\left(t_{1}\right) \leq 0$. If for some constant $M>$ $0,|x(t)| \leq M$ for $t \in\left[\tau^{2}\left(t_{1}\right), t_{1}\right]_{\mathbb{T}}$, then

$$
|x(t)| \leq f^{\dagger}(M)+\lambda \int_{\tau\left(t_{1}\right)}^{t}|r(s)| \Delta s \quad \text { for } t \in\left[\sigma\left(t_{1}\right), \tau^{-1}\left(\sigma\left(t_{1}\right)\right)\right]_{\mathbb{V}} .
$$

Proof. The techniques employed here syncretize and extend ideas from $[13,14]$. We concentrate on the case where $x(t) \geq-M$ for $t \in\left[\tau^{2}\left(t_{1}\right), t_{1}\right]_{\mathbb{T}}$; the case where $x(t) \leq M$ for $t \in\left[\tau^{2}\left(t_{1}\right), t_{1}\right]_{\mathbb{T}}$ is similar and is omitted. Since $x\left(t_{1}\right) x^{\sigma}\left(t_{1}\right) \leq 0$, there exists a real number 
$\xi \in\left[t_{1}-1, t_{1}\right]$ such that

$$
x\left(t_{1}\right)+\left[x^{\sigma}\left(t_{1}\right)-x\left(t_{1}\right)\right]\left(\xi-t_{1}+1\right)=0 .
$$

By (H1), $f^{\dagger}$ is nonnegative and nondecreasing, thus $f(x(t)) \geq-f^{\dagger}(x(t)) \geq-f^{\dagger}(M)$ for $t \in\left[\tau^{2}\left(t_{1}\right), t_{1}\right]_{\mathbb{T}}$. From (1.1), we have

$$
x^{\Delta}(t) \leq p(t) f^{\dagger}(M)+|r(t)|, \quad t \in\left[\tau\left(t_{1}\right), \tau^{-1}\left(t_{1}\right)\right]_{\mathbb{T}},
$$

so that integration and the fundamental theorem yield

$$
x\left(t_{1}\right)-x(\tau(t)) \leq f^{\dagger}(M) \int_{\tau(t)}^{t_{1}} p(s) \Delta s+\int_{\tau(t)}^{t_{1}}|r(s)| \Delta s, \quad t \in\left[t_{1}, \tau^{-1}\left(t_{1}\right)\right]_{\mathbb{T}} .
$$

Using the characterization of $\xi$ in $(2.3)$, we obtain that for $t \in\left[t_{1}, \tau^{-1}\left(t_{1}\right)\right]_{\mathbb{T}}$,

$$
\begin{aligned}
x(\tau(t)) \geq & x\left(t_{1}\right)-f^{\dagger}(M) \int_{\tau(t)}^{t_{1}} p(s) \Delta s-\int_{\tau(t)}^{t_{1}}|r(s)| \Delta s \\
= & -\left[x^{\sigma}\left(t_{1}\right)-x\left(t_{1}\right)\right]\left(\xi-t_{1}+1\right)-f^{\dagger}(M) \int_{\tau(t)}^{t_{1}} p(s) \Delta s-\int_{\tau(t)}^{t_{1}}|r(s)| \Delta s \\
\geq & -f^{\dagger}(M)\left[\left(\xi-t_{1}\right) \int_{t_{1}}^{\sigma\left(t_{1}\right)} p(s) \Delta s+\int_{\tau(t)}^{\sigma\left(t_{1}\right)} p(s) \Delta s\right] \\
& -\left(\xi-t_{1}+1\right) \mu\left(t_{1}\right)\left|r\left(t_{1}\right)\right|-\int_{\tau(t)}^{t_{1}}|r(s)| \Delta s,
\end{aligned}
$$

where we used (2.4) and Theorem 5.4(4) to arrive at the last line. Continuing in this manner, from $(\mathrm{H} 1)$ and the fact that $f^{\dagger}(x)<x$ for positive $x$, we see that

$$
\begin{gathered}
x^{\Delta}(t) \leq p(t) f^{\dagger}\left(f^{\dagger}(M)\left[\left(\xi-t_{1}\right) \int_{t_{1}}^{\sigma\left(t_{1}\right)} p(s) \Delta s+\int_{\tau(t)}^{\sigma\left(t_{1}\right)} p(s) \Delta s\right]\right. \\
\left.\quad+\left(\xi-t_{1}+1\right) \mu\left(t_{1}\right)\left|r\left(t_{1}\right)\right|+\int_{\tau(t)}^{t_{1}}|r(s)| \Delta s\right) \\
\leq p(t) \int_{\tau(t)}^{\sigma\left(t_{1}\right)}\left(f^{\dagger}(M) p(s)+|r(s)|\right) \Delta s \\
\quad-p(t)\left(t_{1}-\xi\right) \mu\left(t_{1}\right)\left(f^{\dagger}(M) p\left(t_{1}\right)+\left|r\left(t_{1}\right)\right|\right)
\end{gathered}
$$

for $t \in\left[t_{1}, \tau^{-1}\left(t_{1}\right)\right]_{\mathbb{T}}$. Now by (H3) and the choice of $\xi$, we know that

$$
0 \leq \zeta:=\left(t_{1}-\xi\right) \int_{t_{1}}^{\sigma\left(t_{1}\right)} p(s) \Delta s+\int_{\sigma\left(t_{1}\right)}^{\tau^{-1}\left(\sigma\left(t_{1}\right)\right)} p(s) \Delta s \leq \lambda,
$$

which we consider in the following two cases. 
4 Forced delay dynamic equation

Case 1. Suppose that $\zeta$ defined in (2.8) satisfies $\zeta \in(0,1)$. For $t \in\left[\sigma\left(t_{1}\right), \tau^{-1}\left(\sigma\left(t_{1}\right)\right)\right]_{\mathbb{T}}$, we have

$$
\begin{aligned}
x(t)= & x^{\sigma}\left(t_{1}\right)+\int_{\sigma\left(t_{1}\right)}^{t} x^{\Delta}(s) \Delta s \\
\stackrel{(2.3)}{=}\left[x^{\sigma}\left(t_{1}\right)-x\left(t_{1}\right)\right]\left(t_{1}-\xi\right)+\int_{\sigma\left(t_{1}\right)}^{t} x^{\Delta}(s) \Delta s & \stackrel{\stackrel{\text { Theorem } 5.4}{=}\left(t_{1}-\xi\right) \mu\left(t_{1}\right) x^{\Delta}\left(t_{1}\right)+\int_{\sigma\left(t_{1}\right)}^{t} x^{\Delta}(s) \Delta s}{\stackrel{(2.7)}{\leq}}\left(t_{1}-\xi\right) \mu\left(t_{1}\right) p\left(t_{1}\right) \int_{\tau\left(t_{1}\right)}^{\sigma\left(t_{1}\right)}\left(f^{\dagger}(M) p(s)+|r(s)|\right) \Delta s \\
& -\left(t_{1}-\xi\right)^{2} \mu\left(t_{1}\right)^{2} p\left(t_{1}\right)\left(f^{\dagger}(M) p\left(t_{1}\right)+\left|r\left(t_{1}\right)\right|\right) \\
& -\left(t_{1}-\xi\right) \mu\left(t_{1}\right)\left(f^{\dagger}(M) p\left(t_{1}\right)+\left|r\left(t_{1}\right)\right|\right) \int_{\sigma\left(t_{1}\right)}^{t} p(s) \Delta s \\
& +\int_{\sigma\left(t_{1}\right)}^{t} p(s)\left(\int_{\tau(s)}^{\sigma\left(t_{1}\right)}\left(f^{\dagger}(M) p(u)+|r(u)|\right) \Delta u\right) \Delta s \\
\leq & f^{\dagger}(M)\left\{\left(t_{1}-\xi\right) \mu\left(t_{1}\right) p\left(t_{1}\right)\left[\int_{\tau\left(t_{1}\right)}^{\sigma\left(t_{1}\right)} p(s) \Delta s-\left(t_{1}-\xi\right) \mu\left(t_{1}\right) p\left(t_{1}\right)\right]\right. \\
& \left.+\int_{\sigma\left(t_{1}\right)}^{t} p(s)\left[\int_{\tau(s)}^{\sigma\left(t_{1}\right)} p(u) \Delta u-\left(t_{1}-\xi\right) \mu\left(t_{1}\right) p\left(t_{1}\right)\right] \Delta s\right\} \\
& +\left(t_{1}-\xi\right) \mu\left(t_{1}\right) p\left(t_{1}\right) \int_{\tau\left(t_{1}\right)}^{\sigma\left(t_{1}\right)}|r(s)| \Delta s+\int_{\sigma\left(t_{1}\right)}^{t} p(s) \int_{\tau(s)}^{\sigma\left(t_{1}\right)}|r(u)| \Delta u \Delta s,
\end{aligned}
$$

where the last inequality follows from simple factoring and the dropping of the negative terms involving $\left|r\left(t_{1}\right)\right|$. Continuing,

$$
\begin{aligned}
& x(t) \stackrel{\text { (H3) }}{\leq} f^{\dagger}(M)\{(\left(t_{1}-\xi\right) \mu\left(t_{1}\right) p\left(t_{1}\right)\left[\lambda-\left(t_{1}-\xi\right) \mu\left(t_{1}\right) p\left(t_{1}\right)\right] \\
&\left.+\int_{\sigma\left(t_{1}\right)}^{\tau^{-1}\left(\sigma\left(t_{1}\right)\right)} p(s)\left[\lambda-\int_{\sigma\left(t_{1}\right)}^{\sigma(s)} p(u) \Delta u-\left(t_{1}-\xi\right) \mu\left(t_{1}\right) p\left(t_{1}\right)\right] \Delta s\right\} \\
&+\left(t_{1}-\xi\right) \int_{t_{1}}^{\sigma\left(t_{1}\right)} p(s) \Delta s \int_{\tau\left(t_{1}\right)}^{\sigma\left(t_{1}\right)}|r(u)| \Delta u+\int_{\sigma\left(t_{1}\right)}^{t} p(s) \int_{\tau(s)}^{\sigma\left(t_{1}\right)}|r(u)| \Delta u \Delta s \\
& \stackrel{(2.8)}{\leq} f^{\dagger}(M)\left\{-\left[\left(t_{1}-\xi\right) \mu\left(t_{1}\right) p\left(t_{1}\right)\right]^{2}-\left(t_{1}-\xi\right) \mu\left(t_{1}\right) p\left(t_{1}\right) \int_{\sigma\left(t_{1}\right)}^{\tau^{-1}\left(\sigma\left(t_{1}\right)\right)} p(s) \Delta s\right. \\
&+\left.+\lambda \zeta-\int_{\sigma\left(t_{1}\right)}^{\tau^{-1}\left(\sigma\left(t_{1}\right)\right)} p(s)\left(\int_{\sigma\left(t_{1}\right)}^{\sigma(s)} p(u) \Delta u\right) \Delta s\right\} \\
&+\left(\int_{t_{1}}^{\tau^{-1}\left(\sigma\left(t_{1}\right)\right)} p(s) \Delta s\right)\left(\int_{\tau\left(t_{1}\right)}^{t}|r(s)| \Delta s\right) .
\end{aligned}
$$


Using Lemma 2.1 on the last double integral involving $p$,

$$
\begin{aligned}
x(t) \leq & f^{\dagger}(M)\left\{-\left[\left(t_{1}-\xi\right) \mu\left(t_{1}\right) p\left(t_{1}\right)\right]^{2}-\left(t_{1}-\xi\right) \mu\left(t_{1}\right) p\left(t_{1}\right) \int_{\sigma\left(t_{1}\right)}^{\tau^{-1}\left(\sigma\left(t_{1}\right)\right)} p(s) \Delta s\right. \\
& \left.+\lambda \zeta-\frac{1}{2}\left(\int_{\sigma\left(t_{1}\right)}^{\tau^{-1}\left(\sigma\left(t_{1}\right)\right)} p(s) \Delta s\right)^{2}-\frac{1}{2} \int_{\sigma\left(t_{1}\right)}^{\tau^{-1}\left(\sigma\left(t_{1}\right)\right)} \mu(s) p(s)^{2} \Delta s\right\} \\
& +\lambda \int_{\tau\left(t_{1}\right)}^{t}|r(s)| \Delta s \\
= & f^{\dagger}(M)\left(\lambda \zeta-\left[\frac{\zeta^{2}}{2}+\frac{\left(\left(t_{1}-\xi\right) \mu\left(t_{1}\right) p\left(t_{1}\right)\right)^{2}}{2}+\int_{\sigma\left(t_{1}\right)}^{\tau^{-1}\left(\sigma\left(t_{1}\right)\right)} \frac{\mu(s)}{2}(p(s))^{2} \Delta s\right]\right) \\
& +\lambda \int_{\tau\left(t_{1}\right)}^{t}|r(s)| \Delta s .
\end{aligned}
$$

Define

$$
m(s):= \begin{cases}\left(t_{1}-\xi\right) \sqrt{\mu(s)} p(s), & s \leq t_{1} \\ \sqrt{\mu(s)} p(s), & s>t_{1}\end{cases}
$$

so that $m$ is right-dense continuous and

$$
x(t) \leq f^{\dagger}(M)\left(\lambda \zeta-\frac{\zeta^{2}}{2}-\frac{1}{2} \int_{t_{1}}^{\tau^{-1}\left(\sigma\left(t_{1}\right)\right)} m^{2}(s) \Delta s\right)+\lambda \int_{\tau\left(t_{1}\right)}^{t}|r(s)| \Delta s
$$

By the Cauchy-Schwarz inequality [4, Theorem 6.15],

$$
\begin{aligned}
& \int_{t_{1}}^{\tau^{-1}\left(\sigma\left(t_{1}\right)\right)} m^{2}(s) \Delta s \\
& \quad \geq \frac{1}{\tau^{-1}\left(\sigma\left(t_{1}\right)\right)-t_{1}}\left(\int_{t_{1}}^{\tau^{-1}\left(\sigma\left(t_{1}\right)\right)} m(s) \Delta s\right)^{2} \\
& \quad=\frac{1}{\tau^{-1}\left(\sigma\left(t_{1}\right)\right)-t_{1}}\left(\left(t_{1}-\xi\right)\left(\mu\left(t_{1}\right)\right)^{3 / 2} p\left(t_{1}\right)+\int_{\sigma\left(t_{1}\right)}^{\tau^{-1}\left(\sigma\left(t_{1}\right)\right)} p(s) \sqrt{\mu(s)} \Delta s\right)^{2} \\
& \quad \stackrel{(1.5)}{\geq} 2\left(\lambda-\frac{3}{2}\right) \zeta^{2} .
\end{aligned}
$$

Thus, for $t \in\left[\sigma\left(t_{1}\right), \tau^{-1}\left(\sigma\left(t_{1}\right)\right)\right]_{\mathbb{T}}$,

$$
x(t) \leq f^{\dagger}(M)\left(\lambda \zeta-\frac{\zeta^{2}}{2}-\left(\lambda-\frac{3}{2}\right) \zeta^{2}\right)+\lambda \int_{\tau\left(t_{1}\right)}^{t}|r(s)| \Delta s
$$


6 Forced delay dynamic equation

If $q(x):=\lambda x-x^{2} / 2-(\lambda-3 / 2) x^{2}$, then $q^{\prime}(0)>0$ and $q^{\prime}(1)=2-\lambda \geq 0$ by the choice of $\lambda$ in (1.5), so that $q$ is increasing on $[0,1]$. Consequently,

$$
x(t) \leq f^{\dagger}(M)+\lambda \int_{\tau\left(t_{1}\right)}^{t}|r(s)| \Delta s, \quad t \in\left[\sigma\left(t_{1}\right), \tau^{-1}\left(\sigma\left(t_{1}\right)\right)\right]_{\mathbb{T}} .
$$

Case 2. Suppose $1 \leq \zeta \leq \lambda$ for $\zeta$ as in (2.8). Actually, from (H3), we have in this case that $\int_{t_{1}}^{\tau^{-1}\left(\sigma\left(t_{1}\right)\right)} p(s) \Delta s \in[1, \lambda]$. Note that

$$
g(t):=\int_{t}^{\tau^{-1}\left(\sigma\left(t_{1}\right)\right)} p(s) \Delta s-1, \quad t \in\left[t_{1}, \tau^{-1}\left(\sigma\left(t_{1}\right)\right)\right]_{\mathbb{T}}
$$

is a delta-differentiable and decreasing function, so that by [4, Theorem 1.16(i)], $g$ is continuous on $t \in\left[t_{1}, \tau^{-1}\left(\sigma\left(t_{1}\right)\right)\right]_{\mathbb{T}}$. Since $g\left(t_{1}\right) \geq 0$ and $g\left(\tau^{-1}\left(\sigma\left(t_{1}\right)\right)\right)=-1<0$, by the intermediate value theorem [4, Theorem 1.115], there exists $t_{2} \in\left[t_{1}, \tau^{-1}\left(\sigma\left(t_{1}\right)\right)\right)_{\mathbb{T}}$ such that either $g\left(t_{2}\right)=0$ or $g\left(t_{2}\right)>0>g^{\sigma}\left(t_{2}\right)$. Either way,

$$
\int_{\sigma\left(t_{2}\right)}^{\tau^{-1}\left(\sigma\left(t_{1}\right)\right)} p(s) \Delta s<1 \leq \int_{t_{2}}^{\tau^{-1}\left(\sigma\left(t_{1}\right)\right)} p(s) \Delta s=\mu\left(t_{2}\right) p\left(t_{2}\right)+\int_{\sigma\left(t_{2}\right)}^{\tau^{-1}\left(\sigma\left(t_{1}\right)\right)} p(s) \Delta s,
$$

ergo there exists a real number $\phi \in\left[t_{2}-1, t_{2}\right)$ such that

$$
\int_{\sigma\left(t_{2}\right)}^{\tau^{-1}\left(\sigma\left(t_{1}\right)\right)} p(s) \Delta s+\left(t_{2}-\phi\right) \mu\left(t_{2}\right) p\left(t_{2}\right)=1
$$

Using (2.3) and (2.4), we have for $t \in\left[t_{1}, t_{2}\right]_{\mathbb{T}}$ that

$$
\begin{aligned}
x(t)= & \left(t_{1}-\xi\right) \mu\left(t_{1}\right) x^{\Delta}\left(t_{1}\right)+\int_{\sigma\left(t_{1}\right)}^{t} x^{\Delta}(s) \Delta s \\
\leq & \left(t_{1}-\xi\right) \mu\left(t_{1}\right)\left(p\left(t_{1}\right) f^{\dagger}(M)+\left|r\left(t_{1}\right)\right|\right)+\int_{\sigma\left(t_{1}\right)}^{t}\left(p(s) f^{\dagger}(M)+|r(s)|\right) \Delta s \\
\leq & f^{\dagger}(M)\left(\left(t_{1}-\xi\right) \mu\left(t_{1}\right) p\left(t_{1}\right)+\int_{\sigma\left(t_{1}\right)}^{t_{2}} p(s) \Delta s\right) \\
& +\left(t_{1}-\xi\right) \mu\left(t_{1}\right)\left|r\left(t_{1}\right)\right|+\int_{\sigma\left(t_{1}\right)}^{t}|r(s)| \Delta s \\
& \leq f^{\dagger}(M) \int_{t_{1}}^{t_{2}} p(s) \Delta s+\int_{t_{1}}^{t}|r(s)| \Delta s<f^{\dagger}(M)+\lambda \int_{\tau\left(t_{1}\right)}^{t}|r(s)| \Delta s,
\end{aligned}
$$


where the last inequality follows from our choice of $t_{2}$. For $t \in\left[\sigma\left(t_{2}\right), \tau^{-1}\left(\sigma\left(t_{1}\right)\right)\right]_{\mathbb{T}}$, with (2.3), we see that

$$
\begin{aligned}
x(t)= & \left(t_{1}-\xi\right) \mu\left(t_{1}\right) x^{\Delta}\left(t_{1}\right)+\int_{\sigma\left(t_{1}\right)}^{t} x^{\Delta}(s) \Delta s \\
= & {\left[\left(t_{1}-\xi\right) \mu\left(t_{1}\right) x^{\Delta}\left(t_{1}\right)+\left(\phi-t_{2}+1\right) \mu\left(t_{2}\right) x^{\Delta}\left(t_{2}\right)+\int_{\sigma\left(t_{1}\right)}^{t_{2}} x^{\Delta}(s) \Delta s\right] } \\
& +\left[\left(t_{2}-\phi\right) \mu\left(t_{2}\right) x^{\Delta}\left(t_{2}\right)+\int_{\sigma\left(t_{2}\right)}^{t} x^{\Delta}(s) \Delta s\right]=S_{1}+S_{2},
\end{aligned}
$$

where $S_{1}$ is the first grouping and $S_{2}$ is the second. Using (2.4) for $S_{1}$ and (2.7) for $S_{2}$,

$$
\begin{aligned}
S_{1} \leq & f^{\dagger}(M)\left(\left(t_{1}-\xi\right) \mu\left(t_{1}\right) p\left(t_{1}\right)+\left(\phi-t_{2}\right) \mu\left(t_{2}\right) p\left(t_{2}\right)+\int_{\sigma\left(t_{1}\right)}^{\sigma\left(t_{2}\right)} p(s) \Delta s\right) \\
& +\left(t_{1}-\xi\right) \mu\left(t_{1}\right)\left|r\left(t_{1}\right)\right|+\left(\phi-t_{2}\right) \mu\left(t_{2}\right)\left|r\left(t_{2}\right)\right|+\int_{\sigma\left(t_{1}\right)}^{\sigma\left(t_{2}\right)}|r(s)| \Delta s, \\
S_{2} \leq & f^{\dagger}(M)\left(t_{2}-\phi\right) \mu\left(t_{2}\right) p\left(t_{2}\right)\left[\int_{\tau\left(t_{2}\right)}^{\sigma\left(t_{1}\right)} p(s) \Delta s-\left(t_{1}-\xi\right) \mu\left(t_{1}\right) p\left(t_{1}\right)\right] \\
& +f^{\dagger}(M) \int_{\sigma\left(t_{2}\right)}^{\tau^{-1}\left(\sigma\left(t_{1}\right)\right)} p(s)\left[\int_{\tau(s)}^{\sigma\left(t_{1}\right)} p(u) \Delta u-\left(t_{1}-\xi\right) \mu\left(t_{1}\right) p\left(t_{1}\right)\right] \Delta s \\
& +\left(t_{2}-\phi\right) \mu\left(t_{2}\right) p\left(t_{2}\right)\left(\int_{\tau\left(t_{2}\right)}^{\sigma\left(t_{1}\right)}|r(s)| \Delta s-\left(t_{1}-\xi\right) \mu\left(t_{1}\right)\left|r\left(t_{1}\right)\right|\right) \\
& +\int_{\sigma\left(t_{2}\right)}^{t} p(s)\left(\int_{\tau(s)}^{\sigma\left(t_{1}\right)}|r(u)| \Delta u-\left(t_{1}-\xi\right) \mu\left(t_{1}\right)\left|r\left(t_{1}\right)\right|\right) \Delta s .
\end{aligned}
$$

Then continuing for $t \in\left[\sigma\left(t_{2}\right), \tau^{-1}\left(\sigma\left(t_{1}\right)\right)\right]_{\mathbb{T}}$ while recalling (2.19), we have

$$
\begin{aligned}
x(t) \leq f^{\dagger}(M)( & {\left[\left(t_{1}-\xi\right) \mu\left(t_{1}\right) p\left(t_{1}\right)+\left(\phi-t_{2}\right) \mu\left(t_{2}\right) p\left(t_{2}\right)+\int_{\sigma\left(t_{1}\right)}^{\sigma\left(t_{2}\right)} p(s) \Delta s\right] } \\
& \times\left[\int_{\sigma\left(t_{2}\right)}^{\tau^{-1}\left(\sigma\left(t_{1}\right)\right)} p(s) \Delta s+\left(t_{2}-\phi\right) \mu\left(t_{2}\right) p\left(t_{2}\right)\right] \\
& +\left(t_{2}-\phi\right) \mu\left(t_{2}\right) p\left(t_{2}\right)\left[\int_{\tau\left(t_{2}\right)}^{\sigma\left(t_{1}\right)} p(s) \Delta s-\left(t_{1}-\xi\right) \mu\left(t_{1}\right) p\left(t_{1}\right)\right]
\end{aligned}
$$


Forced delay dynamic equation

$$
\begin{array}{r}
\left.+\int_{\sigma\left(t_{2}\right)}^{\tau^{-1}\left(\sigma\left(t_{1}\right)\right)} p(s)\left[\int_{\tau(s)}^{\sigma\left(t_{1}\right)} p(u) \Delta u-\left(t_{1}-\xi\right) \mu\left(t_{1}\right) p\left(t_{1}\right)\right] \Delta s\right) \\
+\left(t_{1}-\xi\right) \mu\left(t_{1}\right)\left|r\left(t_{1}\right)\right|+\left(\phi-t_{2}\right) \mu\left(t_{2}\right)\left|r\left(t_{2}\right)\right|+\int_{\sigma\left(t_{1}\right)}^{\sigma\left(t_{2}\right)}|r(s)| \Delta s \\
+\left(t_{2}-\phi\right) \mu\left(t_{2}\right) p\left(t_{2}\right)\left(\int_{\tau\left(t_{2}\right)}^{\sigma\left(t_{1}\right)}|r(s)| \Delta s-\left(t_{1}-\xi\right) \mu\left(t_{1}\right)\left|r\left(t_{1}\right)\right|\right) \\
+\int_{\sigma\left(t_{2}\right)}^{t} p(s)\left(\int_{\tau(s)}^{\sigma\left(t_{1}\right)}|r(u)| \Delta u-\left(t_{1}-\xi\right) \mu\left(t_{1}\right)\left|r\left(t_{1}\right)\right|\right) \Delta s .
\end{array}
$$

Proceeding by rearranging,

$$
\begin{aligned}
x(t) \leq & f^{\dagger}(M)\left(\int_{\sigma\left(t_{2}\right)}^{\tau^{-1}\left(\sigma\left(t_{1}\right)\right)} p(s)\left[\left(\phi-t_{2}\right) \mu\left(t_{2}\right) p\left(t_{2}\right)+\int_{\tau(s)}^{\sigma\left(t_{2}\right)} p(u) \Delta u\right] \Delta s\right. \\
& \left.+\left(t_{2}-\phi\right) \mu\left(t_{2}\right) p\left(t_{2}\right)\left[\left(\phi-t_{2}\right) \mu\left(t_{2}\right) p\left(t_{2}\right)+\int_{\tau\left(t_{2}\right)}^{\sigma\left(t_{2}\right)} p(s) \Delta s\right]\right) \\
& +\left(t_{1}-\xi\right) \mu\left(t_{1}\right)\left|r\left(t_{1}\right)\right| \int_{t}^{\tau^{-1}\left(\sigma\left(t_{1}\right)\right)} p(s) \Delta s+\int_{\sigma\left(t_{2}\right)}^{t} p(s)\left(\int_{\tau(s)}^{\sigma\left(t_{1}\right)}|r(u)| \Delta u\right) \Delta s \\
& +\left(t_{2}-\phi\right) \mu\left(t_{2}\right)\left(p\left(t_{2}\right) \int_{\tau\left(t_{2}\right)}^{\sigma\left(t_{1}\right)}|r(s)| \Delta s-\left|r\left(t_{2}\right)\right|\right)+\int_{\sigma\left(t_{1}\right)}^{\sigma\left(t_{2}\right)}|r(s)| \Delta s .
\end{aligned}
$$

Using (H3) in the first two lines and properties of delta integrals in the last two lines, we arrive at

$$
\begin{aligned}
x(t) \leq & f^{\dagger}(M) \int_{\sigma\left(t_{2}\right)}^{\tau^{-1}\left(\sigma\left(t_{1}\right)\right)} p(s)\left(\left(\phi-t_{2}\right) \mu\left(t_{2}\right) p\left(t_{2}\right)+\lambda-\int_{\sigma\left(t_{2}\right)}^{\sigma(s)} p(u) \Delta u\right) \Delta s \\
& +f^{\dagger}(M)\left(t_{2}-\phi\right) \mu\left(t_{2}\right) p\left(t_{2}\right)\left[\left(\phi-t_{2}\right) \mu\left(t_{2}\right) p\left(t_{2}\right)+\lambda\right] \\
& +\int_{\sigma\left(t_{2}\right)}^{\tau^{-1}\left(\sigma\left(t_{1}\right)\right)} p(s) \int_{\tau(s)}^{\sigma\left(t_{1}\right)}|r(u)| \Delta u \Delta s+\int_{\sigma\left(t_{1}\right)}^{\sigma\left(t_{2}\right)}|r(s)| \Delta s \\
& +\left(\int_{t_{2}}^{\sigma\left(t_{2}\right)} p(s) \Delta s\right)\left(\int_{\tau\left(t_{2}\right)}^{\sigma\left(t_{1}\right)}|r(s)| \Delta s\right) .
\end{aligned}
$$


Applying (2.19) to the terms involving $f^{\dagger}(M)$ and combining some of the remaining integrals, we see that

$$
\begin{aligned}
x(t) \leq & f^{\dagger}(M)\left(\lambda-\int_{\sigma\left(t_{2}\right)}^{\tau^{-1}\left(\sigma\left(t_{1}\right)\right)} p(s) \int_{\sigma\left(t_{2}\right)}^{\sigma(s)} p(u) \Delta u \Delta s-\left[\left(t_{2}-\phi\right) \mu\left(t_{2}\right) p\left(t_{2}\right)\right]^{2}\right. \\
& \left.-\left(t_{2}-\phi\right) \mu\left(t_{2}\right) p\left(t_{2}\right) \int_{\sigma\left(t_{2}\right)}^{\tau^{-1}\left(\sigma\left(t_{1}\right)\right)} p(s) \Delta s\right) \\
& +\left(\int_{t_{2}}^{\tau^{-1}\left(\sigma\left(t_{1}\right)\right)} p(s) \Delta s\right)\left(\int_{\tau\left(t_{2}\right)}^{\sigma\left(t_{1}\right)}|r(s)| \Delta s\right)+\int_{\sigma\left(t_{1}\right)}^{\sigma\left(t_{2}\right)}|r(s)| \Delta s \\
\leq & f^{\dagger}(M)\left(\lambda-\frac{1}{2}-\frac{1}{2} \int_{\sigma\left(t_{2}\right)}^{\tau^{-1}\left(\sigma\left(t_{1}\right)\right)} \mu(s)(p(s))^{2} \Delta s-\frac{1}{2}\left[\left(t_{2}-\phi\right) \mu\left(t_{2}\right) p\left(t_{2}\right)\right]^{2}\right) \\
& +\left(\int_{t_{2}}^{\tau^{-1}\left(\sigma\left(t_{1}\right)\right)} p(s) \Delta s\right)\left(\int_{\tau\left(t_{2}\right)}^{\sigma\left(t_{2}\right)}|r(s)| \Delta s\right)
\end{aligned}
$$

using Lemma 2.1 and (2.19) again to arrive at the first line, and using the choice of $t_{2}$ for the second. Thus, as in (2.15), for $t \in\left[\sigma\left(t_{2}\right), \tau^{-1}\left(\sigma\left(t_{1}\right)\right)\right]_{\mathbb{T}}$,

$$
\begin{aligned}
x(t) & \leq f^{\dagger}(M)\left(\lambda-\frac{1}{2}-\left(\lambda-\frac{3}{2}\right)\right)+\lambda \int_{\tau\left(t_{1}\right)}^{t}|r(s)| \Delta s \\
& =f^{\dagger}(M)+\lambda \int_{\tau\left(t_{1}\right)}^{t}|r(s)| \Delta s .
\end{aligned}
$$

Lemma 2.3. Suppose that (H1)-(H3) hold. Let $x$ be a solution of (1.1) and let $t_{1} \in \mathbb{T}$ be as in Lemma 2.2. Then $x$ is a bounded solution of (1.1).

Proof. The techniques used here are similar to those on $\mathbb{R}$ found in [13]. Let $M:=\max$ $\left\{|x(t)|: t \in\left[\tau^{2}\left(t_{1}\right), t_{1}\right]_{\mathbb{t}}\right\}$. Then by Lemma 2.2,

$$
|x(t)| \leq f^{\dagger}(M)+\lambda \int_{\tau\left(t_{1}\right)}^{t}|r(s)| \Delta s, \quad t \in\left[\sigma\left(t_{1}\right), \tau^{-1}\left(\sigma\left(t_{1}\right)\right)\right]_{\mathbb{T}} .
$$

To prove that $x$ is a bounded solution of (1.1), let

$$
t_{1}^{*}:=\sup \left\{t \in\left[\sigma\left(t_{1}\right), \tau^{-1}\left(\sigma\left(t_{1}\right)\right)\right]_{\mathbb{T}}: x(t) x^{\sigma}(t) \leq 0\right\}
$$

for $n \geq 2$, take

$$
\begin{aligned}
& t_{n}^{\prime}:=\min \left\{t \in\left[\tau^{1-n}\left(\sigma\left(t_{1}\right)\right), \tau^{-n}\left(\sigma\left(t_{1}\right)\right)\right]_{\mathbb{T}}: x(t) x^{\sigma}(t) \leq 0\right\}, \\
& t_{n}^{*}:=\sup \left\{t \in\left[\tau^{1-n}\left(\sigma\left(t_{1}\right)\right), \tau^{-n}\left(\sigma\left(t_{1}\right)\right)\right]_{\mathbb{T}}: x(t) x^{\sigma}(t) \leq 0\right\} .
\end{aligned}
$$


10 Forced delay dynamic equation

If there is no generalized zero in $\left[\tau^{1-n}\left(\sigma\left(t_{1}\right)\right), \tau^{-n}\left(\sigma\left(t_{1}\right)\right)\right]_{\mathbb{T}}$, take

$$
t_{n}^{\prime}:=\tau^{1-n}\left(\sigma\left(t_{1}\right)\right), \quad t_{n}^{*}:=\tau^{-n}\left(\sigma\left(t_{1}\right)\right)
$$

By Lemma 2.2, for $t \in\left[\sigma\left(t_{1}\right), \sigma\left(t_{1}^{*}\right)\right]_{\mathbb{T}}$,

$$
|x(t)| \leq f^{\dagger}(M)+\lambda \int_{\tau\left(t_{1}\right)}^{t}|r(s)| \Delta s \leq M+\lambda \int_{\tau\left(t_{1}\right)}^{\sigma\left(t_{1}^{*}\right)}|r(s)| \Delta s .
$$

If $t_{2}^{\prime} \in\left[\sigma\left(t_{1}^{*}\right), \tau^{-1}\left(\sigma\left(t_{1}^{*}\right)\right)\right]_{\mathbb{T}}$, then

$$
|x(t)| \leq \sup _{t \in\left[\tau^{2}\left(t_{1}^{*}\right), t_{1}^{*}\right]_{\mathbb{T}}}\{|x(t)|\}+\lambda \int_{\tau\left(t_{1}^{*}\right)}^{t}|r(s)| \Delta s
$$

so that

$$
|x(t)| \leq M+\lambda \int_{\tau\left(t_{1}\right)}^{t_{1}^{*}}|r(s)| \Delta s+\lambda \int_{\tau\left(t_{1}^{*}\right)}^{t_{2}^{\prime}}|r(s)| \Delta s, \quad t \in\left[\sigma\left(t_{1}^{*}\right), t_{2}^{\prime}\right]_{\mathbb{T}} .
$$

On the other hand, if $t_{2}^{\prime}>\tau^{-1}\left(\sigma\left(t_{1}^{*}\right)\right)$, then $x$ has constant sign on $\left[\sigma\left(t_{1}^{*}\right), t_{2}^{\prime}\right]_{\mathbb{T}}$. By $(1.1)$ and the fact that $p, x f(x)>0$,

$$
|x(t)| \leq x\left(\tau^{-1}\left(\sigma\left(t_{1}^{*}\right)\right)\right)+\int_{\tau^{-1}\left(\sigma\left(t_{1}^{*}\right)\right)}^{t_{2}^{\prime}}|r(s)| \Delta s, \quad t \in\left[\tau^{-1}\left(\sigma\left(t_{1}^{*}\right)\right), t_{2}^{\prime}\right]_{\mathbb{T}} .
$$

Moreover, as above,

$$
|x(t)| \leq M+\lambda \int_{\tau\left(t_{1}\right)}^{t_{1}^{*}}|r(s)| \Delta s+\lambda \int_{\tau\left(t_{1}^{*}\right)}^{\tau^{-1}\left(\sigma\left(t_{1}^{*}\right)\right)}|r(s)| \Delta s, \quad t \in\left[\sigma\left(t_{1}^{*}\right), \tau^{-1}\left(\sigma\left(t_{1}^{*}\right)\right)\right]_{\mathbb{T}},
$$

so that

$$
\begin{aligned}
|x(t)| & \leq M+\lambda \int_{\tau\left(t_{1}\right)}^{t_{1}^{*}}|r(s)| \Delta s+\lambda \int_{\tau\left(t_{1}^{*}\right)}^{\tau^{-1}\left(\sigma\left(t_{1}^{*}\right)\right)}|r(s)| \Delta s+\int_{\tau^{-1}\left(\sigma\left(t_{1}^{*}\right)\right)}^{t_{2}^{\prime}}|r(s)| \Delta s \\
& \leq M+\lambda \int_{\tau\left(t_{1}\right)}^{t_{1}^{*}}|r(s)| \Delta s+\lambda \int_{\tau\left(t_{1}^{*}\right)}^{t_{2}^{\prime}}|r(s)| \Delta s, \quad t \in\left[\sigma\left(t_{1}^{*}\right), t_{2}^{\prime}\right]_{\mathbb{T}} .
\end{aligned}
$$

Since $t_{2}^{*}-t_{2}^{\prime} \leq \tau^{-2}\left(\sigma\left(t_{1}\right)\right)-\tau^{-1}\left(\sigma\left(t_{1}\right)\right)$, on $\left[t_{2}^{\prime}, t_{2}^{*}\right]_{\mathbb{T}}$ we have

$$
\begin{aligned}
|x(t)| & \leq \sup _{t \in\left[\tau^{2}\left(t_{2}^{\prime}\right), t_{2}^{\prime}\right]_{\pi}}\{|x(t)|\}+\lambda \int_{\tau\left(t_{2}^{\prime}\right)}^{t}|r(s)| \Delta s \\
& \leq M+\lambda \int_{\tau\left(t_{1}\right)}^{t_{1}^{*}}|r(s)| \Delta s+\lambda \int_{\tau\left(t_{1}^{*}\right)}^{t_{2}^{\prime}}|r(s)| \Delta s+\lambda \int_{\tau\left(t_{2}^{\prime}\right)}^{t_{2}^{*}}|r(s)| \Delta s .
\end{aligned}
$$


In the same way for $t \in\left[t_{2}^{*}, t_{3}^{\prime}\right]_{\mathbb{T}}$ as for the case $t \in\left[t_{1}^{*}, t_{2}^{\prime}\right]_{\mathbb{T}}$, we arrive at

$$
\begin{aligned}
|x(t)| & \leq \sup _{t \in\left[\tau^{2}\left(t_{2}^{*}\right), t_{2}^{*}\right]_{\pi}}\{|x(t)|\}+\lambda \int_{\tau\left(t_{2}^{*}\right)}^{t_{3}^{\prime}}|r(s)| \Delta s \\
& \leq M+\lambda\left(\int_{\tau\left(t_{1}\right)}^{t_{1}^{*}}|r(s)| \Delta s+\int_{\tau\left(t_{1}^{*}\right)}^{t_{2}^{\prime}}|r(s)| \Delta s+\int_{\tau\left(t_{2}^{\prime}\right)}^{t_{2}^{*}}|r(s)| \Delta s+\int_{\tau\left(t_{2}^{*}\right)}^{t_{3}^{\prime}}|r(s)| \Delta s\right) \\
& \leq M+2 \lambda \int_{\tau\left(t_{1}\right)}^{t_{2}^{\prime}}|r(s)| \Delta s+2 \lambda \int_{\tau\left(t_{2}^{\prime}\right)}^{t_{3}^{\prime}}|r(s)| \Delta s \\
& \leq M+2 \lambda \int_{\tau\left(t_{1}\right)}^{\tau^{-2}\left(t_{1}\right)}|r(s)| \Delta s+2 \lambda \int_{t_{1}}^{\tau^{-3}\left(t_{1}\right)}|r(s)| \Delta s .
\end{aligned}
$$

For $t \in\left[t_{3}^{\prime}, t_{3}^{*}\right]_{\mathbb{T}}$,

$$
\begin{aligned}
|x(t)| & \leq \sup _{t \in\left[\tau^{2}\left(t_{3}^{\prime}\right), t_{3}^{\prime}\right] \mathbb{\pi}}\{|x(t)|\}+\lambda \int_{\tau\left(t_{3}^{\prime}\right)}^{t_{3}^{*}}|r(s)| \Delta s \\
& \leq M+2 \lambda \int_{\tau\left(t_{1}\right)}^{\tau^{-2}\left(t_{1}\right)}|r(s)| \Delta s+2 \lambda \int_{t_{1}}^{\tau^{-3}\left(t_{1}\right)}|r(s)| \Delta s+\lambda \int_{\tau\left(t_{3}^{\prime}\right)}^{t_{3}^{*}}|r(s)| \Delta s .
\end{aligned}
$$

Consequently, for $t \in\left[t_{3}^{*}, t_{4}^{\prime}\right]_{\mathbb{T}}$,

$$
\begin{aligned}
|x(t)| \leq & \sup _{t \in\left[\tau^{2}\left(t_{3}^{*}\right), t_{3}^{*}\right]_{\tau}}\{|x(t)|\}+\lambda \int_{\tau\left(t_{3}^{*}\right)}^{t_{4}^{\prime}}|r(s)| \Delta s \\
\leq & M+2 \lambda \int_{\tau\left(t_{1}\right)}^{\tau^{-2}\left(t_{1}\right)}|r(s)| \Delta s+2 \lambda \int_{t_{1}}^{\tau^{-3}\left(t_{1}\right)}|r(s)| \Delta s \\
& +\lambda \int_{\tau\left(t_{3}^{\prime}\right)}^{t_{3}^{*}}|r(s)| \Delta s+\lambda \int_{\tau\left(t_{3}^{*}\right)}^{t_{4}^{\prime}}|r(s)| \Delta s \\
\leq & M+2 \lambda \int_{\tau\left(t_{1}\right)}^{\tau^{-2}\left(t_{1}\right)}|r(s)| \Delta s+2 \lambda \int_{t_{1}}^{\tau^{-3}\left(t_{1}\right)}|r(s)| \Delta s+2 \lambda \int_{\tau^{-1}\left(t_{1}\right)}^{\tau^{-4}\left(t_{1}\right)}|r(s)| \Delta s \\
\leq & M+2 \lambda \int_{\tau\left(t_{1}\right)}^{t_{1}}|r(s)| \Delta s+4 \lambda \int_{t_{1}}^{\tau^{-1}\left(t_{1}\right)}|r(s)| \Delta s+6 \lambda \int_{\tau^{-1}\left(t_{1}\right)}^{\tau^{-2}\left(t_{1}\right)}|r(s)| \Delta s \\
& +4 \lambda \int_{\tau^{-2}\left(t_{1}\right)}^{\tau^{-3}\left(t_{1}\right)}|r(s)| \Delta s+2 \lambda \int_{\tau^{-3}\left(t_{1}\right)}^{\tau^{-4}\left(t_{1}\right)}|r(s)| \Delta s .
\end{aligned}
$$


12 Forced delay dynamic equation

Through recursion, for $t \in\left[t_{n}^{*}, t_{n+1}^{\prime}\right]_{\mathbb{T}}$, we obtain

$$
\begin{aligned}
|x(t)| \leq & M+2 \lambda \int_{\tau\left(t_{1}\right)}^{t_{1}}|r(s)| \Delta s+4 \lambda \int_{t_{1}}^{\tau^{-1}\left(t_{1}\right)}|r(s)| \Delta s+6 \lambda \int_{\tau^{-1}\left(t_{1}\right)}^{\tau^{-2}\left(t_{1}\right)}|r(s)| \Delta s \\
& +\cdots+6 \lambda \int_{\tau^{2-n}\left(t_{1}\right)}^{\tau^{1-n}\left(t_{1}\right)}|r(s)| \Delta s+4 \lambda \int_{\tau^{1-n}\left(t_{1}\right)}^{\tau^{-n}\left(t_{1}\right)}|r(s)| \Delta s+2 \lambda \int_{\tau^{-n}\left(t_{1}\right)}^{\tau^{-n-1}\left(t_{1}\right)}|r(s)| \Delta s,
\end{aligned}
$$

and for $t \in\left[t_{n+1}^{\prime}, t_{n+1}^{*}\right]_{\mathbb{T}}$,

$$
\begin{aligned}
|x(t)| & \sup _{t \in\left[\tau^{2}\left(t_{n+1}^{\prime}\right), t_{n+1}^{\prime}\right] \pi}\{|x(t)|\}+\lambda \int_{\tau\left(t_{n+1}^{\prime}\right)}^{t_{n+1}^{*}}|r(s)| \Delta s \\
& \leq M+6 \lambda \int_{\tau\left(t_{1}\right)}^{\tau^{-n-1}\left(t_{1}\right)}|r(s)| \Delta s .
\end{aligned}
$$

Now as both $t_{n}^{\prime}$ and $t_{n}^{*}$ go to infinity as $n$ goes to infinity, by (H2) the solution $x$ must be bounded.

Lemma 2.4. Suppose that (H1)-(H3) hold. Let $x$ be a solution of (1.1) and let $t_{1} \in \mathbb{T}$ be as in Lemma 2.2. Then

$$
|x(t)| \leq f^{\dagger}(B)+\lambda \int_{\tau\left(t_{1}\right)}^{t}|r(s)| \Delta s, \quad t \in\left[\sigma\left(t_{1}\right), \infty\right)_{\mathbb{T}},
$$

where $B:=\sup _{t \geq t_{0}}|x(t)|$.

Proof. By Lemma 2.3, $x$ is a bounded solution of (1.1). Set $B:=\sup _{t \geq t_{0}}|x(t)|$, but suppose that (2.44) is false. Then there exists

$$
T_{1}:=\inf \left\{t>\tau^{-1}\left(\sigma\left(t_{1}\right)\right):|x(t)|>f^{\dagger}(B)+\lambda \int_{\tau\left(t_{1}\right)}^{t}|r(s)| \Delta s\right\} .
$$

Clearly

$$
|x(t)| \leq f^{\dagger}(B)+\lambda \int_{\tau\left(t_{1}\right)}^{t}|r(s)| \Delta s, \quad t \in\left[\sigma\left(t_{1}\right), T_{1}\right)_{\mathbb{T}},
$$

and we have the following cases.

Case 1. (A) Suppose $x\left(T_{1}\right)>f^{\dagger}(B)+\lambda \int_{\mathcal{\tau}\left(t_{1}\right)}^{T_{1}}|r(s)| \Delta s$. By continuity and the choice of $T_{1}$, $T_{1}$ is a left-scattered point with $\left|x\left(\rho\left(T_{1}\right)\right)\right| \leq f^{\dagger}(B)+\lambda \int_{\tau\left(t_{1}\right)}^{\rho\left(T_{1}\right)}|r(s)| \Delta s$ and $x^{\Delta}\left(\rho\left(T_{1}\right)\right)>0$. By $(1.1)$ and $(\mathrm{H} 1), x\left(\tau\left(\rho\left(T_{1}\right)\right)\right)<0$. Set

$$
T_{0}:=\max \left\{t \in\left[\tau\left(\rho\left(T_{1}\right)\right), \rho\left(T_{1}\right)\right)_{\mathbb{T}}: x(t) x^{\sigma}(t) \leq 0\right\} .
$$

Then $x\left(T_{0}\right) x^{\sigma}\left(T_{0}\right) \leq 0$ and $\tau^{2}\left(t_{1}\right) \leq \tau^{3}\left(T_{1}\right) \leq \tau^{2}\left(T_{0}\right) \leq T_{0}<T_{1}$. By $(2.46)$,

$$
|x(t)| \leq f^{\dagger}(B)+\lambda \int_{\tau\left(t_{1}\right)}^{t}|r(s)| \Delta s, \quad t \in\left[\tau^{2}\left(T_{0}\right), T_{0}\right]_{\mathbb{V}} .
$$


Consequently, from Lemma 2.2,

$$
|x(t)| \leq f^{\dagger}(B)+\lambda \int_{\tau\left(t_{1}\right)}^{t}|r(s)| \Delta s \quad \text { on }\left[\sigma\left(T_{0}\right), \tau^{-1}\left(\sigma\left(T_{0}\right)\right)\right]_{\mathbb{T}} .
$$

Since $\tau\left(\rho\left(T_{1}\right)\right) \leq T_{0}<\rho\left(T_{1}\right)$ and $\tau$ is increasing, $\sigma\left(T_{0}\right) \leq T_{1}$ and

$$
f^{\dagger}(B)+\lambda \int_{\tau\left(t_{1}\right)}^{T_{1}}|r(s)| \Delta s<x\left(T_{1}\right) \leq f^{\dagger}(B)+\lambda \int_{\tau\left(t_{1}\right)}^{T_{1}}|r(s)| \Delta s,
$$

a contradiction.

(B) Suppose $x\left(T_{1}\right)=f^{\dagger}(B)+\lambda \int_{\tau\left(t_{1}\right)}^{T_{1}}|r(s)| \Delta s$. Then $T_{1}$ is a right-dense point, $x^{\Delta}\left(T_{1}\right) \geq$ 0 , and there exists $T_{2} \in\left(T_{1}, \tau^{-1}\left(T_{1}\right)\right]_{\mathbb{I}}$ such that $x(t)>f^{\dagger}(B)+\lambda \int_{\tau\left(t_{1}\right)}^{t}|r(s)| \Delta s$ on $\left(T_{1}\right.$, $\left.T_{2}\right]_{\mathbb{T}}$. By $(1.1)$ and $(\mathrm{H} 1), x\left(\tau\left(T_{1}\right)\right) \leq 0$. Set

$$
T_{0}:=\max \left\{t \in\left[\tau\left(T_{1}\right), T_{1}\right)_{\mathbb{T}}: x(t) x^{\sigma}(t) \leq 0\right\} .
$$

Then $x\left(T_{0}\right) x^{\sigma}\left(T_{0}\right) \leq 0$ and $\tau^{2}\left(t_{1}\right) \leq \tau^{3}\left(T_{2}\right) \leq \tau^{2}\left(T_{0}\right) \leq T_{0}<T_{2}$. By $(2.46)$,

$$
|x(t)| \leq f^{\dagger}(B)+\lambda \int_{\tau\left(t_{1}\right)}^{t}|r(s)| \Delta s, \quad t \in\left[\tau^{2}\left(T_{0}\right), T_{0}\right]_{\mathbb{T}} .
$$

As a result, from Lemma 2.2,

$$
|x(t)| \leq f^{\dagger}(B)+\lambda \int_{\tau\left(t_{1}\right)}^{t}|r(s)| \Delta s \quad \text { on }\left[\sigma\left(T_{0}\right), \tau^{-1}\left(\sigma\left(T_{0}\right)\right)\right]_{\mathbb{T}} .
$$

Since $\tau\left(T_{1}\right) \leq T_{0}<T_{2}$ and $\tau$ is increasing, $\sigma\left(T_{0}\right) \leq T_{2} \leq \tau^{-1}\left(\sigma\left(T_{0}\right)\right)$ and

$$
f^{\dagger}(B)+\lambda \int_{\tau\left(t_{1}\right)}^{T_{2}}|r(s)| \Delta s<x\left(T_{2}\right) \leq f^{\dagger}(B)+\lambda \int_{\tau\left(t_{1}\right)}^{T_{2}}|r(s)| \Delta s,
$$

a contradiction.

Case 2. If $x\left(T_{1}\right) \leq-f^{\dagger}(B)-\lambda \int_{\tau\left(t_{1}\right)}^{t}|r(s)| \Delta s$, then (2.46) implies either $x^{\Delta}\left(T_{1}\right) \leq 0$ or $x^{\Delta}\left(\rho\left(T_{1}\right)\right)<0$. Again by (1.1) and (H1), either $x\left(\tau\left(T_{1}\right)\right) \geq 0$ or $x\left(\tau\left(\rho\left(T_{1}\right)\right)\right)>0$. Pick $T_{0}$ as above for either case. Just as above, either case leads to a contradiction.

\section{Solutions of (1.1) go to zero}

We now state our main result on the global asymptotic behavior of solutions of (1.1).

Theorem 3.1. If (H1), (H2), (H3) hold, then every solution of (1.1) goes to zero in the limit. Proof. If $x$ is a nonoscillatory solution of (1.1), assume without loss of generality that $x$ is eventually positive. Then there exist $M>0$ and $T_{0} \geq t_{0}$ such that

$$
0<x(t) \leq\left|x\left(t_{0}\right)\right|+\int_{t_{0}}^{t}|r(s)| \Delta s<M, \quad t \in\left(T_{0}, \infty\right)_{\mathbb{T}} .
$$


14 Forced delay dynamic equation

Suppose that $\liminf _{t \rightarrow \infty} x(t)=2 \varepsilon$ for some $\varepsilon>0$. Pick $T \in\left(\tau^{-1}\left(t_{0}\right), \infty\right)_{\mathbb{T}}$ such that $x(t) \geq \varepsilon$ for $t>\tau(T)$. Since $f$ is continuous and $f(x)>0$ for $x>0, d:=\inf _{\varepsilon \leq x \leq M} f(x)>0$. By (1.1),

$$
x^{\Delta}(t)=-p(t) f(x(\tau(t)))+r(t) \leq-d p(t)+r(t), \quad t \geq T .
$$

Integrating from $T$ to $t$, we see that

$$
x(t) \leq x(T)-d \int_{T}^{t} p(s) \Delta s+\int_{T}^{t}|r(s)| \quad \Delta s \longrightarrow-\infty
$$

as $t \rightarrow \infty$ by (H2) and (H3), a contradiction of $x$ eventually positive. Consequently, $\liminf _{t \rightarrow \infty} x(t)=0$, so there exists an increasing unbounded sequence $\left\{t_{n}\right\}_{n=1}^{\infty}$ in $\mathbb{T}$ such that $\lim _{n \rightarrow \infty} x\left(t_{n}\right)=0$. Let $M^{\prime}:=\limsup _{t \rightarrow \infty} x(t)$. Again there exists a sequence $\left\{t_{n}^{\prime}\right\}_{n=1}^{\infty}$ in $\mathbb{}$ with $t_{n}^{\prime} \geq t_{n}$ such that $\lim _{n \rightarrow \infty} x\left(t_{n}^{\prime}\right)=M^{\prime}$. Using (H2) and the fact that $x^{\Delta}(t) \leq r(t)$,

$$
0<x\left(t_{n}^{\prime}\right) \leq x\left(t_{n}\right)+\int_{t_{n}}^{t_{n}^{\prime}}|r(s)| \quad \Delta s \longrightarrow 0, n \longrightarrow \infty .
$$

Hence $M^{\prime}=0$ and $x$ goes to zero.

Now let $x$ be an oscillatory solution of (1.1). By Lemma 2.4, (2.44) holds. By the oscillatory nature of $x$, there exists a sequence $\left\{t_{n}^{*}\right\}$ in $\mathbb{T}$ such that

$$
x\left(t_{n}^{*}\right) x^{\sigma}\left(t_{n}^{*}\right) \leq 0, \quad \tau\left(t_{1}^{*}\right) \geq \tau^{-1}\left(t_{0}\right), \quad \tau\left(t_{n+1}^{*}\right)>\tau^{-1}\left(t_{n}^{*}\right) .
$$

As in [13], we consider the discrete sequence $\left\{X_{n}\right\}$ given by

$$
X_{1}=B:=\sup _{t \geq t_{0}}|x(t)|, \quad X_{n+1}=f^{\dagger}\left(X_{n}\right)+\lambda \int_{\tau\left(t_{n}^{*}\right)}^{\infty}|r(s)| \Delta s .
$$

Just as in the proof of Lemma 2.3, we arrive at

$$
\sup _{t \in\left[\tau^{2}\left(t_{n}^{*}\right), t_{n}^{*}\right]_{\mathbb{T}}}|x(t)| \leq X_{n}, \quad \sup _{t \geq \sigma\left(t_{n}^{*}\right)}|x(t)| \leq X_{n+1} .
$$

Note that

$$
\sum_{n=1}^{\infty} \lambda \int_{\tau\left(t_{n}^{*}\right)}^{\infty}|r(s)| \Delta s \leq \sum_{n=0}^{\infty} \lambda \int_{\tau^{1-n}\left(t_{1}^{*}\right)}^{\infty}|r(s)| \Delta s \leq \lambda \sum_{n=0}^{\infty} \int_{\tau^{1-n}\left(t_{1}^{*}\right)}^{\infty}|r(s)| \Delta s ;
$$

by $(\mathrm{H} 2)$,

$$
\sum_{n=1}^{\infty} \lambda \int_{\tau\left(t_{n}^{*}\right)}^{\infty}|r(s)| \Delta s=: \sum_{n=1}^{\infty} b_{n}<\infty
$$

Since $X_{n}$ satisfies the difference equation $X_{n+1}=f^{\dagger}\left(X_{n}\right)+b_{n}$, using [13, Lemma 2.3] we have that $X_{n}$ goes to zero as $n \rightarrow \infty$. By the choice of $X_{n}$, the solution $x$ of (1.1) satisfies $\lim _{t \rightarrow \infty} x(t)=0$. 
Corollary 3.2. If (H1) and (H3) hold, then every solution of the unforced equation

$$
x^{\Delta}(t)+p(t) f(x(\tau(t)))=0, \quad t \in\left[t_{0}, \infty\right)_{\mathbb{T}},
$$

goes to zero in the limit.

Remark 3.3. The results of this paper could easily be modified to show that every solution of

$$
x^{\Delta}(t)=\ominus p(t) f(x(\tau(t)))-\ominus r(t), \quad t \in\left[t_{0}, \infty\right)_{\mathbb{T}}, \quad t_{0} \geq 0,
$$

goes to zero in the limit, for appropriately adjusted hypotheses (H1), (H2), (H3), where $\ominus p(t):=-p(t) /(1+\mu(t) p(t))$ for $t \in \mathbb{T}$ and $p \in \mathscr{R}$ (see Definition 5.5).

\section{Forced delay equation on isolated time scales}

Let $\mathbb{T}$ be a time scale unbounded above, with every point both left and right scattered, and consider the food-limited population model [7, 13] given by the delay differential equation

$$
y^{\prime}(t)=p y(t) \frac{N-y(t-\tau)}{N+c p y(t-\tau)}
$$

where $y$ is the population density, $p>0$ is a constant growth rate, $N>0$ is the carrying capacity of the habitat, $\tau>0$ is the time delay, and $c>0$ is constant. From this we obtain the following modified equation:

$$
\frac{1}{y(t)} \frac{d y(t)}{d t}=p \frac{N-y(\lfloor t\rfloor-\lfloor\tau\rfloor)}{N+c p y(\lfloor t\rfloor-\lfloor\tau\rfloor)},
$$

where $\lfloor t\rfloor:=\sup \{s \in \mathbb{T}: s \leq t\}$ is the "time-scale" part of the continuous variable $t$. On any interval of the form $[s, \sigma(s)$ ), integrate (4.2) from $s$ to $t$ to obtain for $s \leq t<\sigma(s)$ that

$$
y(t)=y(s) \exp \left(p \frac{N-y(s-\lfloor\tau\rfloor)}{N+c p y(s-\lfloor\tau\rfloor)}(t-s)\right) .
$$

Replacing $t$ by $\sigma(s)$,

$$
y^{\sigma}(s)=y(s) \exp \left(p \mu(s) \frac{N-y(s-\lfloor\tau\rfloor)}{N+c p y(s-\lfloor\tau\rfloor)}\right)
$$

Note that if $\mathbb{Z}=\mathbb{Z}$ and $\lfloor\tau\rfloor=0$, then $\sigma(s)=s+1$ and $\mu(s) \equiv 1$, and this is the simple genotype selection model suggested in [9] and [11, Exercise 1.18(6)]. Thus for any isolated time scale $\mathbb{T}$ that is unbounded above, we consider

$$
\frac{y^{\sigma}(t)}{y(t)}=\exp \left(p \mu(t) \frac{N-y(\tau(t))}{N+c p y(\tau(t))}+r(t)\right)
$$


16 Forced delay dynamic equation

for some delay $\tau: \mathbb{T} \rightarrow \mathbb{T}$ and some function $r: \mathbb{T} \rightarrow \mathbb{R}$ satisfying (H2). In (4.5), let $y=$ $N e^{x}, y^{\sigma}=N e^{x \circ \sigma}$, and $y \circ \tau=N e^{x \circ \tau}$ to obtain

$$
x^{\Delta}(t)=-p \frac{e^{x(\tau(t))}-1}{1+c p e^{x(\tau(t))}}+\frac{r(t)}{\mu(t)} .
$$

If

$$
f(x):=\frac{e^{x}-1}{1+c p e^{x}}
$$

then $f$ is continuous with $x f(x)>0$ for $x \neq 0$ and $f(0)=0$. As shown in [13], if $c p>1 / 3$, then $|f(x)|<|x|$ for $x \neq 0$ as well.

Theorem 4.1. Suppose $c p>1 / 3$ and $\mathbb{T}$ is an isolated time scale with $t_{0} \in \mathbb{T}$. If

$$
\begin{gathered}
\sum_{n=0}^{\infty} \sum_{t \in\left[\tau^{1-n}\left(t_{0}\right), \infty\right)_{\mathbb{T}}}|r(t)|<\infty, \\
p(\sigma(t)-\tau(t)) \leq \lambda \quad \forall t \in\left[t_{0}, \infty\right)_{\mathbb{T}},
\end{gathered}
$$

then every positive solution of (4.5) goes to $N$ in the limit.

Proof. Let $y$ be a positive solution of (4.5). As above, the substitution $y=N e^{x}$ makes $x$ a solution of (4.6). Since $c p>1 / 3,[13$, Theorem 3.1] shows that (H1) is satisfied. To check $(\mathrm{H} 2)$, note that on isolated time scales,

$$
\sum_{n=0}^{\infty} \int_{\tau^{1-n}\left(t_{0}\right)}^{\infty} \frac{|r(s)| \Delta s}{\mu(s)}=\sum_{n=0}^{\infty} \sum_{t \in\left[\tau^{1-n}\left(t_{0}\right), \infty\right) \mathbb{T}}|r(t)|<\infty
$$

by assumption. In the same way, (H3) is satisfied for constant $p>0$, as

$$
\int_{\tau(t)}^{\sigma(t)} p(s) \Delta s=p(\sigma(t)-\tau(t)) \leq \lambda, \quad t \in\left[t_{0}, \infty\right)_{\mathbb{T}} .
$$

Hence by Theorem 3.1, every solution $x$ of (4.6) goes to zero in the limit. But then every positive solution $y=N e^{x}$ of (4.5) goes to $N$.

Example 4.2. Let $\mathbb{T}=h \mathbb{Z}$ for some $h \in(0,1), c>0$, and let $\tau(t):=t-h k$ for $t \in \mathbb{T}$ and $k \in \mathbb{N}$. If

$$
\begin{aligned}
& \sum_{t=-k}^{\infty} t|r(t h)|<\infty, \\
& \frac{1}{3 c}<p \leq \frac{3 k+4}{2 h(k+1)^{2}},
\end{aligned}
$$

then every positive solution of (4.5) goes to $N$ in the limit. 
Proof. Observe that $\lambda=(3 k+4) / 2(k+1)$, and $\sigma(t)-\tau(t)=h(k+1)$. Now we show that (4.12) is equivalent to (4.8) on $h \mathbb{Z}$. In fact, both will be shown to be equivalent to

$$
\sum_{t=-k}^{\infty} \sum_{s=t}^{\infty}|r(s h)|<\infty
$$

the idea of these three equivalences is adapted from the real case found in [10, Lemma 3.3]. First note that (4.8), (4.12), and (4.14) all imply that $\sum_{t=-k}^{\infty}|r(t h)|<\infty$. To see that (4.8) implies (4.14), we switch the order of summing in (4.14) to get that

$$
\begin{aligned}
\sum_{t=-k}^{\infty} \sum_{s=t}^{\infty}|r(s h)| & =\sum_{n=0}^{\infty} \sum_{t=(n-1) k}^{n k-1} \sum_{s=t}^{\infty}|r(s h)| \\
& =\sum_{n=0}^{\infty}\left(\sum_{t=(n-1) k}^{n k-1}(t-(n-1) k+1)|r(t h)|+k \sum_{t=n k}^{\infty}|r(t h)|\right)
\end{aligned}
$$

As a result,

$$
\begin{aligned}
\sum_{n=0}^{\infty} \sum_{t=n k}^{\infty}|r(t h)| & \leq \frac{1}{k} \sum_{t=-k}^{\infty} \sum_{s=t}^{\infty}|r(s h)|=\frac{1}{k} \sum_{n=0}^{\infty} \sum_{t=(n-1) k}^{n k-1} \sum_{s=t}^{\infty}|r(s h)| \\
& \leq \frac{1}{k} \sum_{n=0}^{\infty} \sum_{t=(n-1) k}^{n k-1} \sum_{s=(n-1) k}^{\infty}|r(s h)|=\sum_{n=0}^{\infty} \sum_{t=(n-1) k}^{\infty}|r(t h)| .
\end{aligned}
$$

Therefore (4.8) implies (4.14). And since

$$
\sum_{t=-k}^{\infty} \sum_{s=t}^{\infty}|r(s h)|=\sum_{s=-k}^{\infty} \sum_{t=-k}^{s}|r(s h)|=\sum_{t=-k}^{\infty}(t+1-k)|r(t h)|,
$$

(4.12) implies (4.14). Therefore (4.8) implies (4.12). Thus all the hypotheses of Theorem 4.1 are met.

\section{Appendix on time scales}

The definitions below merely serve as a preliminary introduction to the time-scale calculus; they can be found in the context of a much more robust treatment than is allowed here in the textbooks $[4,5]$ and the references therein.

Definition 5.1. Define the forward (backward) jump operator $\sigma(t)$ at $t$ for $t<\sup \mathbb{\mathbb { T }}$ (resp., $\rho(t)$ at $t$ for $t>\inf \mathbb{T})$ by

$$
\sigma(t)=\inf \{\tau>t: \tau \in \mathbb{T}\}, \quad(\rho(t)=\sup \{\tau<t: \tau \in \mathbb{T}\}), \quad \forall t \in \mathbb{T} .
$$

Also define $\sigma(\sup \mathbb{T})=\sup \mathbb{T}$ if $\sup \mathbb{T}<\infty$, and $\rho(\inf \mathbb{\mathbb { V }})=\inf \mathbb{\mathbb { H }}$ if inf $\mathbb{T}>-\infty$. Define the graininess function $\mu: \mathbb{T} \rightarrow \mathbb{R}$ by $\mu(t)=\sigma(t)-t$. 
Throughout this work, the assumption is made that $\mathbb{T}$ is unbounded above and has the topology that it inherits from the standard topology on the real numbers $\mathbb{R}$. Also assume throughout that $a<b$ are points in $\mathbb{T}$ and define the time-scale interval $[a, b]_{\mathbb{T}}=\{t \in$ $\mathbb{T}: a \leq t \leq b\}$. The jump operators $\sigma$ and $\rho$ allow the classification of points in a time scale in the following way: if $\sigma(t)>t$, the point $t$ is right-scattered, while if $\rho(t)<t$, then $t$ is left-scattered. If $\sigma(t)=t$, the point $t$ is right-dense; if $t>\inf \mathbb{T}$ and $\rho(t)=t$, then $t$ is left-dense.

Definition 5.2. Fix $t \in \mathbb{T}$ and let $y: \mathbb{T} \rightarrow \mathbb{R}$. Define $y^{\Delta}(t)$ to be the number (if it exists) with the property that given $\epsilon>0$, there is a neighborhood $U$ of $t$ such that for all $s \in U$,

$$
\left|[y(\sigma(t))-y(s)]-y^{\Delta}(t)[\sigma(t)-s]\right| \leq \epsilon|\sigma(t)-s|
$$

Call $y^{\Delta}(t)$ the (delta) derivative of $y$ at $t$.

Definition 5.3. If $F^{\Delta}(t)=f(t)$, then define the (Cauchy) delta integral by

$$
\int_{a}^{t} f(s) \Delta s=F(t)-F(a) .
$$

The following theorem is due to Hilger [8].

Theorem 5.4. Assume that $f: \mathbb{T} \rightarrow \mathbb{R}$ and let $t \in \mathbb{T}$.

(1) If $f$ is differentiable at then $f$ is continuous at $t$.

(2) If $f$ is continuous at $t$ and $t$ is right-scattered, then $f$ is differentiable at $t$ with

$$
f^{\Delta}(t)=\frac{f(\sigma(t))-f(t)}{\sigma(t)-t} .
$$

(3) If $f$ is differentiable and $t$ is right-dense, then

$$
f^{\Delta}(t)=\lim _{s \rightarrow t} \frac{f(t)-f(s)}{t-s}
$$

(4) If $f$ is differentiable at $t$, then $f(\sigma(t))=f(t)+\mu(t) f^{\Delta}(t)$.

Next we define the important concept of right-dense continuity. An important fact concerning right-dense continuity is that every right-dense continuous function has a delta antiderivative [4, Theorem 1.74]. This implies that the delta definite integral of any right-dense continuous function exists.

Definition 5.5. A function $f: \mathbb{T} \rightarrow \mathbb{R}$ is right-dense continuous (denoted by $f \in C_{\mathrm{rd}}(\mathbb{T} ; \mathbb{R})$ ) provided that $f$ is continuous at every right-dense point $t \in \mathbb{T}$, and $\lim _{s \rightarrow t^{-}} f(s)$ exists and is finite at every left-dense point $t \in \mathbb{T}$. A function $p$ is regressive provided that $1+$ $\mu(t) p(t) \neq 0$ for all $t \in \mathbb{T}$, and

$$
\mathscr{R}:=\left\{p \in C_{\mathrm{rd}}(\mathbb{T} ; \mathbb{R}): 1+\mu(t) p(t) \neq 0, t \in \mathbb{T}\right\}
$$




\section{References}

[1] D. R. Anderson, Asymptotic behavior of solutions for neutral delay dynamic equations on time scales, Advances in Difference Equations 2006 (2006), Article ID 80850, 11 pages.

[2] D. R. Anderson and J. Hoffacker, Positive periodic time-scale solutions for functional dynamic equations, The Australian Journal of Mathematical Analysis and Applications 3 (2006), no. 1, $1-14$, article 5.

[3] D. R. Anderson, R. J. Krueger, and A. C. Peterson, Delay dynamic equations with stability, Advances in Difference Equations 2006 (2006), Article ID 94051, 19 pages.

[4] M. Bohner and A. Peterson, Dynamic Equations on Time Scales, An Introduction with Applications, Birkhäuser Boston, Massachusetts, 2001.

[5] M. Bohner and A. Peterson (eds.), Advances in Dynamic Equations on Time Scales, Birkhäuser Boston, Massachusetts, 2003.

[6] L. H. Erbe, H. Xia, and J. S. Yu, Global stability of a linear nonautonomous delay difference equation, Journal of Difference Equations and Applications 1 (1995), no. 2, 151-161.

[7] K. Gopalsamy, M. R. S. Kulenović, and G. Ladas, Environmental periodicity and time delays in a "food-limited" population model, Journal of Mathematical Analysis and Applications 147 (1990), no. 2, 545-555.

[8] S. Hilger, Analysis on measure chains - a unified approach to continuous and discrete calculus, Results in Mathematics 18 (1990), no. 1-2, 18-56.

[9] V. L. Kocić and G. Ladas, Global Behavior of Nonlinear Difference Equations of Higher Order with Applications, Mathematics and Its Applications, vol. 256, Kluwer Academic, Dordrecht, 1993.

[10] Q. Kong, Y. Sun, and B. Zhang, Nonoscillation of a class of neutral differential equations, Computers \& Mathematics with Applications 44 (2002), no. 5-6, 643-654.

[11] M. R. S. Kulenović and O. Merino, Discrete Dynamical Systems and Difference Equations with Mathematica, Chapman \& Hall/CRC, Florida, 2002.

[12] H. Matsunaga, R. Miyazaki, and T. Hara, Global attractivity results for nonlinear delay differential equations, Journal of Mathematical Analysis and Applications 234 (1999), no. 1, 77-90.

[13] C. Qian and Y. Sun, Global attractivity of solutions of nonlinear delay differential equations with a forcing term, to appear in Nonlinear Analysis.

[14] X. Zhang and J. Yan, Global asymptotic behavior of nonlinear difference equations, Computers \& Mathematics with Applications 49 (2005), no. 9-10, 1335-1345.

Douglas R. Anderson: Department of Mathematics and Computer Science, Concordia College, Moorhead, MN 56562, USA

E-mail address: andersod@cord.edu 